\title{
Modulation of electromagnetic fields by a depolarizer of random polarizer array
}

\author{
Ma, Ning; Hanson, Steen Grüner; Wang, Wei
}

Published in:

Optics Letters

Link to article, DOI:

10.1364/OL.41.001997

Publication date:

2016

Document Version

Peer reviewed version

Link back to DTU Orbit

Citation (APA):

Ma, N., Hanson, S. G., \& Wang, W. (2016). Modulation of electromagnetic fields by a depolarizer of random polarizer array. Optics Letters, 41(9), 1997-2000. https://doi.org/10.1364/OL.41.001997

\section{General rights}

Copyright and moral rights for the publications made accessible in the public portal are retained by the authors and/or other copyright owners and it is a condition of accessing publications that users recognise and abide by the legal requirements associated with these rights.

- Users may download and print one copy of any publication from the public portal for the purpose of private study or research.

- You may not further distribute the material or use it for any profit-making activity or commercial gain

- You may freely distribute the URL identifying the publication in the public portal

If you believe that this document breaches copyright please contact us providing details, and we will remove access to the work immediately and investigate your claim. 


\title{
Modulation of electromagnetic fields by a depolarizer of random polarizer array
}

\author{
Ning MA ${ }^{1}$, Steen G. HANSON ${ }^{2}$, AND WeI WANG ${ }^{1, *}$ \\ ${ }^{1}$ Institute of Photonics and Quantum Sciences, School of Engineering and Physical Sciences, Heriot-Watt University, Edinburgh, Eh14 4AS, UK \\ ${ }^{2}$ Department of Photonics Engineering, DTU Fotonik, P.O. Box 49, DK-4000 Roskilde, Denmark \\ *Corresponding author: W.Wang@hw.ac.uk
}

Received XX Month XXXX; revised XX Month, XXXX; accepted XX Month XXXX; posted XX Month XXXX (Doc. ID XXXXX); published XX Month XXXX

\begin{abstract}
The statistical properties of the electric fields with random change of polarization state in space generated by a depolarizer are investigated on the basis of the coherence matrix. The depolarizer is a polarizer array composed of a multitude of contiguous square cells of polarizers with randomly distributed orientation angles whereand the incident fields experience the - a random polarization modulation after passing through the depolarizer. The propagation of the modulated electric fields through any quadratic optical system is examined within the frame work of the complex ABCD matrix to show how the degree of coherence and polarization changes on propagation. (C) 2015 Optical Society of America
\end{abstract}

OCIS codes: (030.1640) Coherence; (030.6600) Statistical optics; (230.5440) Polarization-selective devices; (260.5430) Polarization

http://dx.doi.org/10.1364/AO.99.099999

Optical systems are often sensitive to the polarization states of the incident light. In many applications, the polarization sensitivity may cause serious errors in the system's output if some unwanted polarization of the inputs hasve been introduced [1]. To reduce the undesirable effects of the polarization sensitivity, a depolarizer has been widely used to scramble the polarization state of incident beam. An ideal depolarizer can convert a polarized beam into-a light with temporally and/or spatially random polarization states. Unfortunately, all commercially available depolarizers can only provide the output light with pseudo-random polarization states having a spatially, temporally or spectrally periodic variations. To overcome this difficulty of pseudo-randomness, many different approaches have been proposed to minimize the unwanted effects of polarization sensitivity in optical system by using a large number of small polarizer cells with their optical axes randomly oriented [2-5]. Although different patterned structures for crystalline chips of birefringent materials have been proposed to achieve the depolarization effect, neither the physical models for such devices nor the theoretical analysis of their modulation to the incident electric fields have been given to understand their performance as the $\underline{a}$ depolarizer.
In this letter, we present a model for the depolarizer composed of a multitude of contiguous square cells of linear polarizers with randomly orientated polarizing axes. Within the framework of the complex ABCD method, the statistical properties of electric fields modulated by the depolarizer are investigated to observe their evolutions of the spatial degree of polarization and the degree of spatial coherence during propagation.

As a specific example of a depolarizer, we consider the random polarizer array illustrated in Fig. 1. This structure consists of a multitude of contiguous $l \times l$ square cells. In each cell, the polarization orientation indicated by the small arrow has been randomly and independently chosen so that only the incident vector wave component parallel to the direction of the local arrow can pass. The random polarizer array itself may be regarded as being infinite in extent, although only a finite portion lies within the pupil of the optical system. Because of a lack of knowledge of the exact location of the depolarizer on the scale of a single cell, an-the assumption has been taken-made that is at least the statistical properties of the structure is wide-sense stationary over space. The location of the depolarizer with respect to the optical axis is chosen to be random with a uniform distribution of probability over any square cell.

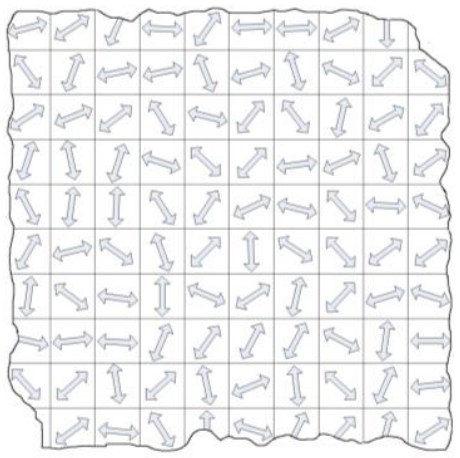

Figure 1. Diagram of depolarizer: random polarizer array

In order to specify the statistical properties of the electric fields $\mathbf{E}(\mathbf{r})=\left\{\tilde{E}_{x}(\mathbf{r}), \tilde{E}_{y}(\mathbf{t}\}\right.$ during its propagation, a $2 \times 2$ coherence matrix is given by [6-7] 


$$
\mathbf{W}\left(\mathbf{r}_{1}, \mathbf{r}_{2}\right)=\left(\begin{array}{ll}
\left\langle\tilde{E}_{x}^{*}\left(\mathbf{r}_{1}\right) \tilde{E}_{x}\left(\mathbf{r}_{2}\right)\right\rangle & \left\langle\tilde{E}_{x}^{*}\left(\mathbf{r}_{1}\right) \tilde{E}_{y}\left(\mathbf{r}_{2}\right)\right\rangle \\
\left\langle\tilde{E}_{y}^{*}\left(\mathbf{r}_{1}\right) \tilde{E}_{x}\left(\mathbf{r}_{2}\right)\right\rangle & \left\langle\tilde{E}_{y}^{*}\left(\mathbf{r}_{1}\right) \tilde{E}_{y}\left(\mathbf{r}_{2}\right)\right\rangle
\end{array}\right),
$$

where angular brackets $\langle\ldots\rangle$ indicates the-an ensemble average, and the asterisk * denotes the complex conjugate. Based on the coherence matrix, the degree of coherence $\eta$ and the degree of polarization $P$ could be calculated. They areare given by

$$
\eta\left(\mathbf{r}_{1}, \mathbf{r}_{2}\right)=\frac{\operatorname{tr} \mathbf{W}\left(\mathbf{r}_{1}, \mathbf{r}_{2}\right)}{\sqrt{\operatorname{tr} \mathbf{W}\left(\mathbf{r}_{1}, \mathbf{r}_{1}\right) \operatorname{tr} \mathbf{W}\left(\mathbf{r}_{2}, \mathbf{r}_{2}\right)}},
$$

and

$$
P(\mathbf{r})=\left\{1-\frac{4 \operatorname{det} \mathbf{W}(\mathbf{r}, \mathbf{r})}{[\operatorname{tr} \mathbf{W}(\mathbf{r}, \mathbf{r})]^{2}}\right\}^{1 / 2},
$$

where $t r$ and det depict the trace and determinant of the matrix, respectively.

When the incident electric field passes through the depolarizer, the polarization state is modified accordingly, i.e., $\mathbf{E}^{t}(\mathbf{r})=\mathbf{T}(\mathbf{r}) \mathbf{E}^{i}(\mathbf{r})$ with the superscript $i$ or $t$ representing the incident or transmission field. With reference to the depolarizer pattern of Fig. 1, each square cell is a polarizer with its polarization angle $\varphi$ to the $\hat{x}$ axis and the transmission matrix (Jones matrix) of the device is [8]

$$
\begin{aligned}
\mathbf{T}(\mathbf{r}) & =\left(\begin{array}{cc}
T_{x x}(\mathbf{r}) & T_{x y}(\mathbf{r}) \\
T_{y x}(\mathbf{r}) & T_{y y}(\mathbf{r})
\end{array}\right) \\
& =\left(\begin{array}{cc}
\cos ^{2} \varphi(\mathbf{r}) & \sin \varphi(\mathbf{r}) \cos \varphi(\mathbf{r}) \\
\cos \varphi(\mathbf{r}) \sin \varphi(\mathbf{r}) & \sin ^{2} \varphi(\mathbf{r})
\end{array}\right) .
\end{aligned}
$$

Let $\mathbf{W}^{i}\left(\mathbf{r}_{1}, \mathbf{r}_{2}\right)$ be the coherence matrix of the incident beam, then the coherence matrix $\mathbf{W}^{t}\left(\mathbf{r}_{1}, \mathbf{r}_{2}\right)$ of the modulated field just behind the depolarizer is given [6]

$$
\mathbf{W}^{t}\left(\mathbf{r}_{1}, \mathbf{r}_{2}\right)=\left\langle\mathbf{T}^{\dagger}\left(\mathbf{r}_{1}\right) \mathbf{W}^{i}\left(\mathbf{r}_{1}, \mathbf{r}_{2}\right) \mathbf{T}\left(\mathbf{r}_{2}\right)\right\rangle,
$$

where the $\uparrow$ denotes the Hermitian conjugate.

Since different cells have statistically independent polarization angle for each linear polarizer, the coherence matrix of the modulate fields can be written

$$
\begin{aligned}
\mathbf{W}^{t}\left(\mathbf{r}_{1}, \mathbf{r}_{2}\right) & ={ }^{S} \mathbf{W}^{t}\left(\mathbf{r}_{1}, \mathbf{r}_{2}\right) \operatorname{Prob}\left\{\begin{array}{l}
\mathbf{r}_{1} \text { and } \mathbf{r}_{2} \text { are } \\
\text { in the same cell }
\end{array}\right\} \\
& +{ }^{D} \mathbf{W}^{t}\left(\mathbf{r}_{1}, \mathbf{r}_{2}\right) \operatorname{Prob}\left\{\begin{array}{l}
\mathbf{r}_{1} \text { and } \mathbf{r}_{2} \text { are } \\
\text { in different cells }
\end{array}\right\} .
\end{aligned}
$$

In the expression above, ${ }^{S} \mathbf{W}^{t}$ and ${ }^{D} \mathbf{W}^{t}$ have been introduced to denote the coherence matrices for the transmitted fields with points $\mathbf{r}_{1}$ and $\mathbf{r}_{2}$ falling into the same and different cells, respectively. Similar to the random checkerboard absorbing screen [9-10], we are able to write the desired probabilities because of the uniform distribution of the absolute location of the depolarizer. They are

$$
\operatorname{Prob}\left\{\mathbf{r}_{1}, \mathbf{r}_{2} \text { in the same cell }\right\}=\wedge\left(\Delta r_{x} / l\right) \wedge\left(\Delta r_{y} / l\right),
$$

$$
\operatorname{Prob}\left\{\begin{array}{c}
\mathbf{r}_{1} \text { and } \mathbf{r}_{2} \text { are in } \\
\text { different cells }
\end{array}\right\}=1-\wedge\left(\Delta r_{x} / l\right) \wedge\left(\Delta r_{y} / l\right),
$$

where $\Delta r_{x}$ and $\Delta r_{y}$ are the two components of the vector $\Delta \mathbf{r}=\mathbf{r}_{1}-\mathbf{r}_{2}$ along $\hat{x}$ and $\hat{y}$ directions, and the unit triangle function $\wedge(x)=1-|x|$ for $|x| \leq 1$ and zero otherwise.

Notice that the axis of polarizer in each cell is assumed random and independent from cell to cell. We have

$$
{ }^{S} \mathbf{W}^{t}\left(\mathbf{r}_{1}, \mathbf{r}_{2}\right)=\left(\begin{array}{ll}
{ }^{S} W_{x x}^{t}\left(\mathbf{r}_{1}, \mathbf{r}_{2}\right) & { }^{S} W_{x y}^{t}\left(\mathbf{r}_{1}, \mathbf{r}_{2}\right) \\
{ }^{S} W_{y x}^{t}\left(\mathbf{r}_{1}, \mathbf{r}_{2}\right) & { }^{S} W_{y y}^{t}\left(\mathbf{r}_{1}, \mathbf{r}_{2}\right)
\end{array}\right),
$$

(8)

with

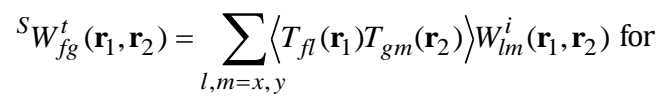

$(f, g=x, y)$, and

(9)

$$
{ }^{D} \mathbf{W}^{t}\left(\mathbf{r}_{1}, \mathbf{r}_{2}\right)=\left(\begin{array}{cc}
{ }^{D} W_{x x}^{t}\left(\mathbf{r}_{1}, \mathbf{r}_{2}\right) & { }^{D} W_{x y}^{t}\left(\mathbf{r}_{1}, \mathbf{r}_{2}\right) \\
{ }^{D} W_{y x}^{t}\left(\mathbf{r}_{1}, \mathbf{r}_{2}\right) & { }^{D} W_{y y}^{t}\left(\mathbf{r}_{1}, \mathbf{r}_{2}\right)
\end{array}\right) .
$$

with ${ }^{D} W_{f g}^{t}\left(\mathbf{r}_{1}, \mathbf{r}_{2}\right)=\sum_{l, m=x, y}\left\langle T_{f l}\left(\mathbf{r}_{1}\right)\right\rangle\left\langle T_{g m}\left(\mathbf{r}_{2}\right)\right\rangle W_{l m}^{i}\left(\mathbf{r}_{1}, \mathbf{r}_{2}\right)$.

When Eqs. (8) and (9) have been derived, we have made use of the fact that the coherence property of the electric fields and correlation property of the random polarizer array are statistically independent and the ensemble average denoted by angular brackets has been taken over the depolarizer. The coherence matrix for the modulated field just behind the depolarizer can be assessed only if some specific assumptions are made regarding the statistics of the polarization angle $\varphi$ for the random polarizer array. A case of the-most interest is the depolarizer whose stochastic polarization angle has a uniform probability distribution function within $[0, \pi)$. Under the assumption of $p_{\varphi}(\varphi)=1 / \pi$, the first-order and second-order moments of the elements of Jones Matrix in Eq. (4) have been evaluated and listed in the Table 1.

Table 1. First-order and Second-order Moments of the Elements in Jones Matrix

$$
\begin{array}{cc}
\left\langle T_{x x}\right\rangle=\left\langle\cos ^{2} \varphi(\mathbf{r})\right\rangle=1 / 2 & \left\langle T_{y y}\right\rangle=\left\langle\sin ^{2} \varphi(\mathbf{r})\right\rangle=1 / 2 \\
\left\langle T_{x y}\right\rangle=\langle\cos \varphi(\mathbf{r}) \sin \varphi(\mathbf{r})\rangle=0 & \left\langle T_{y x}\right\rangle=\langle\cos \varphi(\mathbf{r}) \sin \varphi(\mathbf{r})\rangle=0 \\
\left\langle T_{x x}^{2}\right\rangle=\left\langle\cos ^{4} \varphi(\mathbf{r})\right\rangle=3 / 8 & \left\langle T_{y y}^{2}\right\rangle=\left\langle\sin ^{4} \varphi(\mathbf{r})\right\rangle=3 / 8 \\
\left\langle T_{x y}^{2}\right\rangle=\left\langle T_{y x}^{2}\right\rangle=\left\langle\sin ^{2} \varphi(\mathbf{r}) \cos ^{2} \varphi(\mathbf{r})\right\rangle=1 / 8 \\
\left\langle T_{x x} T_{x y}\right\rangle=\left\langle T_{x x} T_{y x}\right\rangle=\left\langle\sin \varphi(\mathbf{r}) \cos ^{3} \varphi(\mathbf{r})\right\rangle=0 \\
\left\langle T_{x y} T_{y y}\right\rangle=\left\langle T_{y x} T_{y y}\right\rangle=\left\langle\sin ^{3} \varphi(\mathbf{r}) \cos \varphi(\mathbf{r})\right\rangle=0 \\
\left\langle T_{x x} T_{y y}\right\rangle=\left\langle\sin ^{2} \varphi(\mathbf{r}) \cos ^{2} \varphi(\mathbf{r})\right\rangle=1 / 8
\end{array}
$$$$
\left\langle T_{y y}\right\rangle=\left\langle\sin ^{2} \varphi(\mathbf{r})\right\rangle=1 / 2
$$

In this case, the coherence matrix $\mathbf{W}^{t}$ is now easily found by substituting Eqs. (7)-(9) into Eq (6), with the result

$$
\begin{aligned}
\mathbf{W}^{t}\left(\mathbf{r}_{1}, \mathbf{r}_{2}\right) & =\frac{1}{4}\left(\begin{array}{ll}
W_{x x}^{i} & W_{x y}^{i} \\
W_{y x}^{i} & W_{y y}^{i}
\end{array}\right) \\
& +\frac{1}{8} \wedge\left(\frac{\Delta r_{x}}{l}\right) \wedge\left(\frac{\Delta r_{y}}{l}\right)\left(\begin{array}{cc}
W_{x x}^{i}+W_{y y}^{i} & -W_{x y}^{i}+W_{y x}^{i} \\
-W_{y x}^{i}+W_{x y}^{i} & W_{y y}^{i}+W_{x x}^{i}
\end{array}\right) .
\end{aligned}
$$


(10)

Equation (10) provides us with the desired correlation properties of the modulated electric fields passing through the depolarizer of the random polarizer array and can be considered as one of the prime result of this letter.

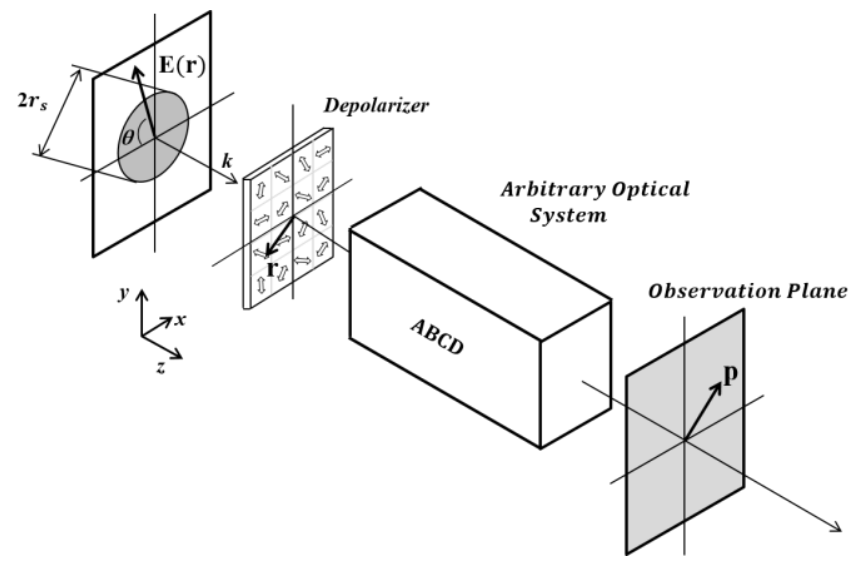

Figure 2. Schematic of the setup for obtaining the degree of polarization and coherence of a field propagating through a random polarizer array plate and an ABCD optical systems

Figure. 2 shows the optical system to examine the propagation of the electric field modulated by a random polarizer array. An incident electric field with its wave vector $\vec{k}$ is illustrated-incident on the depolarizer of -random polarizer array. The modulated light passing through the optical system arrives at the observation plane. To understand the statistical properties of the modulated electric fields, we need to calculate the propagation of the mutual coherence matrix through an optical system. For demonstration purpose only, and without loss of generality, we will perform our calculations only for an incident field that is a linearly polarized, spatially coherent Gaussian beam with the electric field making an angle $\theta$ with the $\hat{x}$ axis. The corresponding coherence matrix for such a beam just in front of the depolarizer is

$$
\mathbf{W}^{i}\left(\mathbf{r}_{1}, \mathbf{r}_{2}\right)=I_{o} \exp \left\{-\frac{\left|\mathbf{r}_{1}\right|^{2}+\left|\mathbf{r}_{2}\right|^{2}}{r_{s}^{2}}\right\}\left(\begin{array}{cc}
\cos ^{2} \theta & \cos \theta \sin \theta \\
\cos \theta \sin \theta & \sin ^{2} \theta
\end{array}\right),
$$

where $I_{0}$ and $r_{s}$ are the on-axis intensity and the beam width of the incident field, respectively. On substituting from Eq. (11) into Eq. (10), the expression for the mutual coherence matrix of the modulated beam just behind the depolarizer is

$$
\begin{aligned}
& \mathbf{W}^{o}\left(\mathbf{r}_{1}, \mathbf{r}_{2}\right)=\frac{I_{o}}{8} \exp \left\{-\frac{\left|\mathbf{r}_{1}\right|^{2}+\left|\mathbf{r}_{2}\right|^{2}}{r_{s}^{2}}\right\} \\
& \left.\times\left\{\begin{array}{cc}
\cos ^{2} \theta & \sin \theta \cos \theta \\
\sin \theta \cos \theta & \sin ^{2} \theta
\end{array}\right)+\wedge\left(\frac{\Delta r_{x}}{l}\right) \wedge\left(\frac{\Delta r_{y}}{l}\right)\left(\begin{array}{ll}
1 & 0 \\
0 & 1
\end{array}\right)\right\} .
\end{aligned}
$$

Our interest here is the static spatial statistical properties of the modulated electric fields [11-12]. After replacing the conventional time average by spatial average for the ensemble average in Eq. (1) and substituting from Eq. (12) into Eq. (3), we have the spatial degree of polarization for the modulated fields just after the random polarizer array. That is

$$
P^{t}(\mathbf{r})=1 / 2,
$$

which is a constant no matter where the observation point is chosen. As expected, a perfectly polarized incident beam has become spatially partially polarized after passing through the static depolarizer. On substitution from Eq. (12) into Eq. (2), the degree of spatial coherence of the modulated electric fields behind the depolarizer is obtained

$$
\eta^{t}\left(\mathbf{r}_{1}, \mathbf{r}_{2}\right)=0.5\left[1+\wedge\left(\Delta r_{x} / l\right) \wedge\left(\Delta r_{y} / l\right)\right]
$$

indicating a spatially partial coherence has been achieved. Meanwhile, it's also interesting to notice from Eqs. (13) and (14) that both the degree of polarization and the degree of coherence are independent of the polarization angle $\theta$ of the incident beam.

Under the paraxial approximation, the mutual coherence matrix $\mathbf{W}^{o}$ at the observation plane after propagating through a complex-valued $\mathrm{ABCD}$ optical system is given in general terms of the Huygens-Fresnel integral formulation [13-14]

$$
\mathbf{W}^{o}\left(\mathbf{p}_{1}, \mathbf{p}_{2}\right)=\iint \mathbf{W}^{t}\left(\mathbf{r}_{1}, \mathbf{r}_{2}\right) G^{*}\left(\mathbf{p}_{1}, \mathbf{r}_{1}\right) G\left(\mathbf{p}_{2}, \mathbf{r}_{2}\right) d \mathbf{r}_{1} d \mathbf{r}_{2},
$$

where the Green's function is given by

$$
G(\mathbf{p}, \mathbf{r})=-\frac{j k}{2 \pi B} \exp \left\{-\frac{j k}{2 B}\left(A|\mathbf{r}|^{2}-2 \mathbf{r} \cdot \mathbf{p}+D|\mathbf{p}|^{2}\right)\right\}
$$

In the equation above, $j$ is the imaginary unit, $k$ is the wave-number, $\mathrm{A}, \mathrm{B}$, and D are the elements of $\mathrm{ABCD}$ matrix for the whole optical system under consideration, which is determined by the multiplication of the matrices for all the individual optical components, i.e., the lenses, free space propagations and apertures. As an example, we will consider a typical case of free-space propagation over a distance- $z$, whereand the corresponding $\mathrm{ABCD}$ matrix is given by

$$
\hat{M}=\left(\begin{array}{cc}
1-j z / z_{R} & z \\
-j / z_{R} & 1
\end{array}\right)
$$

where $z_{R}=k r_{s}^{2} / 2$ is the Rayleigh range.

By substituting the elements of the ABCD matrix into Eq. (16), one can calculate the free space propagation of the mutual coherence matrix and study the spatial evolutions of the degree of polarization and the degree of coherence in any observation plane $z>0$ in the case where the random polarizer array is illuminated by a linearly polarized Gaussain electromagnetic beam. To present some numerical examples, we have taken the following parameters: $r_{s}=1 \mathrm{~mm}, l=0.1 \mathrm{~mm}$, $\theta=\pi / 4$ and $\lambda=0.633 \mu \mathrm{m}$.

Figure 3 shows the degree of polarization of the electric field modulated by the depolarizer for the-free-space propagation, plotted against the normalized propagation distance $z / z_{R}$ and normalized lateral distance measured in spot size $p / r_{s}$. As expected, the degree of polarization changes appreciably depending both on the propagation distance $z$ and the observation position $\mathbf{p}-$. Instead of a uniform distribution for the modulated electric fields just behind the depolarizer, the degree of polarization for the modulated electric fields after propagation does not remain uniform.

Figure 4 gives the absolute values of the degree of coherence of the modulated electric fields at two positions $\Delta \mathbf{p}=\mathbf{p}_{1}-\mathbf{p}_{2}$ located symmetrically with respect to the $\hat{z}$ axis along the normalized 
propagation distance. It can readily be seen that the degree of coherence takes a large value close to unity for two points located near the optical axis. These figures indicate how the spatial degree of polarization and the degree of spatial coherence evolve for the selected polarization angle of the incident beam.

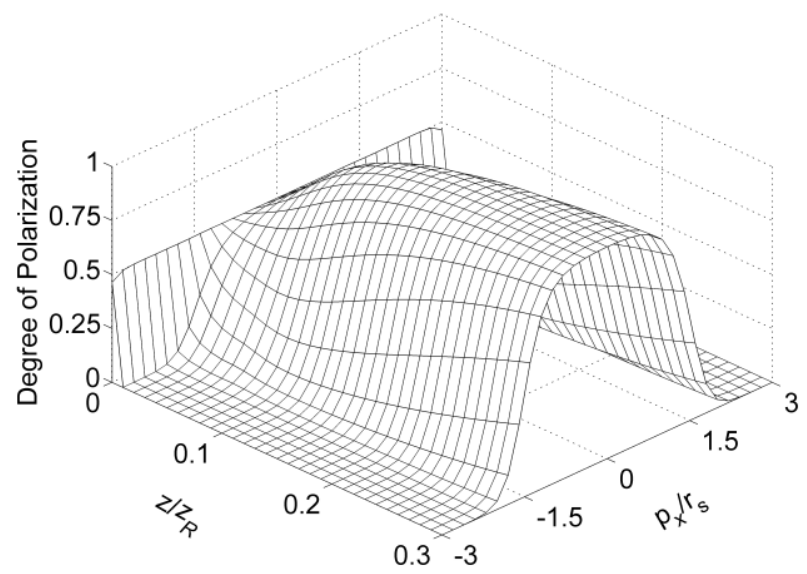

Figure 3. The degree of polarization for free-space propagation as a function of the lateral position measured in spot size units and the propagation distance measured in units of the Rayleigh length.

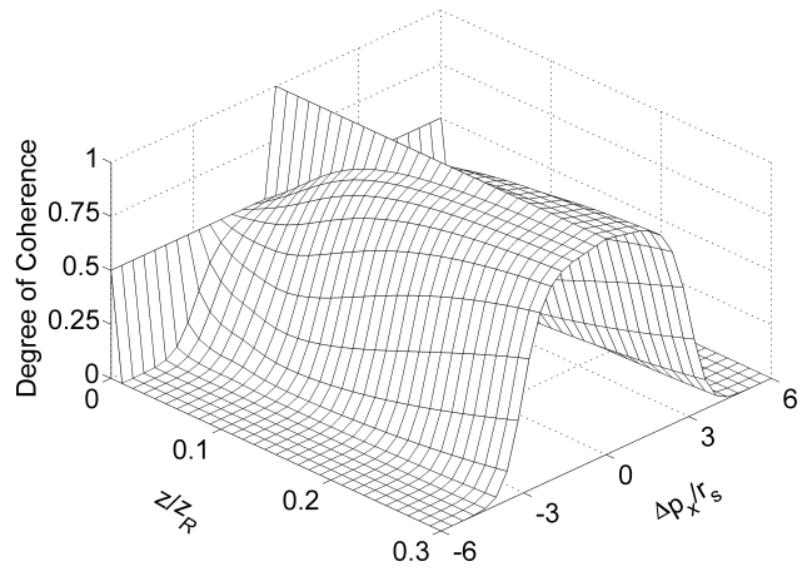

Figure 4. Degree of coherence for free-space propagation at two lateral points located symmetrically.

In summary, we have proposed, to the best of our knowledge, an analytic model for the random polarizer array and examined its performance as a depolarizer to scramble the polarization states of the incident beam. Within the framework of the complex ABCD method, we have studied the evolution of the degree of polarization and the degree of coherence for the electric fields modulated by such depolarizer on propagation in free space. In particular, we have shown that the spatial degree of polarization and the degree of spatial coherence for the modulated fields just behind the random polarizer array are independent of the incident polarization angle. Further, a systematic analysis of the depolarization effect introduced by the random polarizer array will facilitate our the design and optimization of such devices as the a depolarizer, and open up new opportunities of various applications.
Fundings Engineering and Physical Sciences Research Council (EPSRC) (EP/K03643/1); Scottish Universities Physics Alliance (SUPA) (SSG040).

Acknowledgement N. Ma gratefully acknowledge his scholarship supports from Nelson Fund to support his study in UK.

\section{References}

1. W. A. Shurcliff, Polarized Light: Production and Use (Harvard Univ. Press, 1962).

2. J. Begner, "Depolariser," GB2088078 (A), (September 24, 1980).

3. G. S. M. Anthony and S. Maurice, "Depolariser with random orientated regions of polarisation state modulating material," UK Patent 2384865 A (2002).

4. H. Sato and A. Koyanagi, "Depolarization element," JP2008226405, (September 25, 2008).

5. S. Mceldowney, J. Zieba, and M. Newell, "Passive depolariser," EP1892544, (February 27, 2008).

6. L. Mandel and E. Wolf, Optical Coherence and Quantum Optics, (Cambridge Univ. Press 1995).

7. E. Wolf, Introduction to the Theory of Coherence and Polarization of Light (Cambridge University Press, 2007).

8. R. C. Jones, "A new calculus for the treatment of optical systems i. description and discussion of the calculus," J. Opt. Soc. Am. 31, 488-493 (1941).

9. J. W. Goodman, Statistical Optics (Wiley, 2000).

10. E. L. O'Neill, Introduction to Statistical Optics (Addison-Wesley, 1965).

11. W. Wang, S. G. Hanson, and M. Takeda, "Statistics of polarization speckle: theory versus experiment," Proc. SPIE 7388,1-9 (2009).

12. M. Takeda, W. Wang, D. N. Naik, and R. K. Singh, "Spatial statistical optics and spatial correlation holography: a review," Opt. Rev. 21, 849861 (2014)

13. A. E. Siegman, Lasers (University Science Books, 1986).

14. H. T. Yura and S. G. Hanson, "Optical beam wave propagation through complex optical systems," J. Opt. Soc. Am. A, 4, 1931-1948 (1987). 